Revista General de Información y Documentación ISSN: 1132-1873

http://dx.doi.org/10.5209/RGID.56563

\title{
Evaluación de la Usabilidad del Repositorio Institucional de la Universidad de Brasília
}

\author{
Luísa Chaves Café ${ }^{1}$; Ivette Kafure Muñoz ${ }^{2}$
}

Recibido: 8 noviembre 2016 / Aceptado: 3 demayo de 2017

Resumen. Se presenta una investigación, sobre la usabilidad del Repositorio Institucional de la Universidad de Brasília (RIUnB). El objetivo general de la investigación fue describir la interacción entre los usuarios estudiantes de posgrado de la Universidad de Brasília (UnB) y el RIUnB durante el proceso de recuperación de la información. Para alcanzar el objetivo, fue necesario identificar el perfil y el modelo mental de los usuarios, las dificultades de uso durante la recuperación de la información como también, fue caracterizada la interfaz. Fue realizado un estudio de caso por medio de un cuestionario, la evaluación heurística y la observación participante. Los resultados señalan diversos problemas de usabilidad, siendo los más severos relacionados con al sistema de búsqueda. Se concluye que el RIUnB es un servicio de información con potencial para servir las necesidades de información de usuarios, pero es indispensable la inclusión de funcionalidades y corrección de problemas para facilitar la interacción con la interfaz del repositorio. Esta investigación fue presentada en el Programa de Posgrado en Ciencia de la Información de la Universidad de Brasília como requisito para la obtención del título de Maestría en Ciencia de la Información.

Palabras clave: Acceso Abierto; Biblioteca Digital; Comunicación Científica; Estudio de Usuarios; Interacción Humano-Computador; Modelo Mental; Recuperación de la Información; Repositorio Institucional de la Universidad de Brasília; Usabilidad.

\section{[en] Usability Evaluation of the Institutional Repository of Brasília's University}

\begin{abstract}
1 Facultad de Ciencia de la Información. Universidad de Brasília, Brasil.
E-mail: lccafe@gmail.com

2 Facultad de Ciencia de la Información. Universidad de Brasília, Brasil.

E-mail: ivettek@unb.br
\end{abstract}

Abstract. Presents research on information retrieval in the Institutional Repository of the University of Brasilia. The research question is: how does the interaction between postgraduates users and the repository of the University of Brasilia occurs? The research's overall objective is to describe the interaction between users and the Repository during the process of information retrieval. To achieve the overall objective, it was necessary to identify the profile of RIUnB's users, to characterize the repository interface, to identify the mental model of RIUnB's users, and to identify possible difficulties of use of the interface during the information retrieval process. It was developed through a case study using online survey, participant observation and heuristic evaluation. The results show that the participants are master and doctoral students from different fields of knowledge and mainly from 25 to 35 years old. The students frequently research scientific literature in the Internet, but they rarely access repositories. They access RIUnB through Google to research an specific document, a subject 
or an author. The heuristic evaluation indicates has the interface has different usability problems, the most severe are related to the search system. During the observations it was found that users change their perceptions about the repository during the execution of research tasks, but have difficulties during the research process. Concludes that the interaction between graduate students and the RIUnB allows users to retrieve documents, but with difficulties. Therefore, the RIUnB is an information service that may attend the informational needs from its users. However, it is necessary to insert functions and improve usability to facilitate the interaction with the repositorie's interface.

Keywords: Digital Library; Human-Computer Interaction; Information Retrieval; Open access; Scientific communication; User study.

Sumario. 1. Introducción. 2. Revisión de Literatura. 3. Metodología. 4. Resultados. 5. Conclusiones. 6. Referencias bibliográficas.

Cómo citar: Chaves Café, L., Kafure Muñoz, I. (2017) Evaluación de la Usabilidad del Repositorio Institucional de la Universidad de Brasília. Estudio de caso, en Revista General de Información y Documentación, 27 (1), 87-106.

Reconocimientos. Los autores de este trabajo reconocen el apoyo de la Coordenação de Aperfeiçoamento de Pessoal de Nível Superior (CAPES) en el desarrollo de esta investigación.

\section{Introducción}

Los repositorios institucionales son sistemas de información compuestos por colecciones digitales, que son desarrollados a partir de servicios de gestión relacionados a la colecta, organización, diseminación y preservación de la producción académica de los miembros de una institución (Costa, 2014). Estos sistemas pueden ser considerados como un tipo de bibliotecas digitales (Leite, 2009) y actualmente son canales importantes para mediar investigaciones en acceso abierto (Farias, Galindo, 2017). Por lo tanto, es imprescindible ampliar el conocimiento relacionado a las necesidades y expectativas de los usuarios de repositorios y para que el uso de estos sistemas sea eficiente, eficaz y satisfactorio.

El Repositorio Institucional de la Universidad de Brasília (RIUnB) es un repositorio que ha tenido altos índices de consultas y descargas. En 2015, fueron realizadas 3.756.076 descargas de los documentos del repositorio (RIUnB, 2016). El repositorio también es reconocido por su visibilidad y contenido en el ranking de Repositorios de Webometrics, que lo clasifica en el cuarto (40) lugar entre los repositorios brasileños. El RIUnB fue desarrollado en 2004 con el software DSpace a partir del trabajo conjunto entre la Biblioteca Central de la Universidad de Brasília (UnB) y la Facultad de Ciencia de la Información de la UnB (Freitas, Silva y Guimarães, 2009). El DSpace es un software libre creado en 2002 por la iniciativa de las bibliotecas del Massachussetts Institute of Technology (MIT), con el objetivo de auxiliar en la creación de repositorios dinámicos para la producción intelectual disponible en formatos digitales (Souza, Barbosa, Silva, 2017) .

El RIUnB posee diferentes tipos de usuarios, entre ellos estudiantes de pregrado y posgrado, profesores y la comunidad en general que hace parte o no de la UnB. En esta investigación se estudia la usabilidad desde del punto de vista del usuario estudiante de posgrado de la UnB. Se entiende que estos usuarios son 
investigadores en formación. De esa manera, es posible que utilicen los sistemas de información científica con más frecuencia y específicamente, para la búsqueda de la información. Para la recolección de dados de esta investigación, se estudiaron usuarios que no están acostumbrados a utilizar un sistema de información. Y, este tipo de usuarios, pueden ser una fuente de información interesante, debido a la posibilidad de observar en cuanto tiempo y con qué facilidad aprenden a utilizar su interfaz (Norman, 2008). Ello es diferente que estudiar los usuarios que están habituados a utilizar el repositorio, los cuales pueden saber si el RIUnB satisface sus necesidades de información, en la recuperación de la producción científica de su propia institución.

El objetivo general de esta investigación es fue describir la interacción entre estudiantes de posgrado y el Repositorio Institucional de la Universidad de Brasília durante el proceso de recuperación de la información. Para alcanzar este objetivo, fueron determinados cuatro objetivos específicos:

- Identificar el perfil de los usuarios estudiantes de posgrado del RIUnB.

- Describir las características de la interfaz del RIUnB.

- Identificar el modelo mental de los usuarios al respecto del RIUnB.

- Identificar las posibles dificultades de uso de la interfaz del RIUnB en el proceso de recuperación de la información.

Después de alcanzar los objetivos establecidos y describir la interacción entre los usuarios y el Repositorio, fue posible, en términos de usabilidad, el observar si la interacción ocurre de forma eficaz, eficiente y satisfactoria.

\section{Revisión de literatura}

\subsection{Comunicación Científica y Repositorios Digitales}

Es vital para la existencia de la ciencia, una comunicación eficaz, para el desarrollo $\mathrm{y}$ avance de nuevas técnicas y métodos que rubriquen mejorar la eficiencia y eficacia de los procesos de comunicación científica, que son responsabilidad de diversos profesionales dentro de la comunidad (Meadows, 1999). Actualmente la comunicación científica presenta nuevos paradigmas, debido a los avances de las tecnologías de la información. Hurd (2004) afirmó que las tecnologías, principalmente el computador personal y la Internet, causaron cambios en la forma de comunicación entre los investigadores y hoy en día tienen papeles fundamentales en los procesos de comunicación científica. De acuerdo con la autora, las interfaces de los sistemas digitales deben ser eficaces para los investigadores que utilizan estos servicios y por eso deben ser llevados en consideración en la estructuración de los sistemas por los profesionales de la información (Machado, Vianna, 2016). .

Además de las transformaciones tecnológicas, el aumento de los precios de los periódicos colaboraron en el surgimiento del movimiento del acceso abierto a la 
información científica (Swan, 2006). Este movimiento defiende que las publicaciones científicas deben estar disponibles a todos (Bégault, 2009) para lectura, descarga, copia, distribución y búsqueda (Leite, 2009). Como afirma Harnad y Brody (2004), la literatura que trata del acceso abierto tiene mayor potencial para lectores y usuarios, pues permite que los individuos sin vínculos institucionales, que no pueden pagar por los costos de periódicos para que puedan acceder a la literatura científica. De esta forma, la literatura en acceso abierto posee mayor uso e impacto. Suber (2013) destaca que el acceso abierto respeta prácticas de revisión por pares y es compatible con las leyes de derechos de autor, la preservación de documentos impresos, la calidad de la investigación, la indexación en bases de datos y otros atributos asociados a la literatura académica convencional.

Una de las estrategias para garantizar el acceso abierto a la información científica comprende el archivo de la producción científica en repositorios digitales de acceso abierto. Estos sistemas garantizan una mayor visibilidad de la producción científica y la mejor gestión del conocimiento institucional o temático.

\subsection{Interacción Humana con el Computador}

La Interacción Humana con el Computador (IHC) es un área interdisciplinar que posee contribuciones de la Computación, de la Psicología (Egert, Gershon, Van Der Veer, 2012) de la Ciencia de la Información, (Lazzarin, 2012) entre otras áreas del conocimiento. Esta área se preocupa con el diseño, evaluación e implementación de sistemas computacionales interactivos para el uso humano. Así, como en el estudio de los principales fenómenos de uso (Hewett, 1996; Sivaji, Nielsen, Clemmensen, 2017).

La IHC se muestra como un área fundamental para éste estudio, pues la atención inadecuada a los usuarios y al uso de los sistemas conllevan a interfaces de mala calidad y coloca la existencia del sistema en riesgo (Dix, 2004). De acuerdo a esta afirmación, los sistemas que presentan muchas dificultades a sus usuarios pueden dejar de ser usados por su poca usabilidad y, por lo tanto, dejan de existir, aunque tengan el suficiente potencial para satisfacer necesidades de su público-objetivo. Para tratar problemas de interacción y desarrollar interfaces de calidad, la IHC tiene como uno de sus tópicos centrales la usabilidad.

La usabilidad se refiere a la calidad que caracteriza el uso de los programas y aplicaciones. Ella auxilia en la presentación de la interfaz de acuerdo con el perfil de sus usuarios (Cybis, Betiol, Faust, 2010). La norma ISO 9241-11 [17], que trata de orientaciones para la elaboración de interfaces de computadores, define la usabilidad como "el grado en que un producto es usado por los usuarios específicos para alcanzar objetivos específicos con eficacia, eficiencia y satisfacción en un contexto de uso específico". Se observa a partir de la definición, de la usabilidad, se relaciona con la conclusión de un trabajo - eficacia - a partir de un proceso simple y sin demoras - eficiencia - y que durante y al final del procedimiento, el usuario se sienta bien - satisfacción. De esta manera, las necesidades de información del usuario son atendidas y sus objetivos alcanzados. 
Un aspecto mental de usuarios que se muestra relevante para estudios ergonómicos y de Interacción Humana con el Computador es el modelo mental. El modelo mental de un usuario representa el conocimiento del usuario en relación con el cómo utilizar un sistema y sobre cómo funciona este sistema (Rogers, Sharp, Preece, 2013). El modelo mental puede ser analizado a partir de las necesidades y expectativas de un usuario sobre un sistema (Kafure, 2004) y a partir del análisis de la tarea y de la actividad del usuario (Kafure, 2004). De acuerdo con la autoraos autores, la tarea se refiere a lo que el usuario pretende hacer y espera que acontezca al realizar un trabajo. La actividad es la realización del trabajo, comprendiendo sus acciones y decisiones para alcanzar sus objetivos. Al realizar el análisis de las tareas a partir de las perspectivas de los usuarios, se examina la confrontación del modelo mental de la tarea con la actividad (Kafure, 2004).

\subsection{Estudios de Usuarios y el Uso de la Información}

De acuerdo con Björk (2007), existen diversos agentes en la comunicación científica, entre ellos: los investigadores, los financiadores de investigación, los editores, las bibliotecas, las bases de datos, los ejecutores de las investigaciones y los lectores. Estos últimos son definidos como aquellos que buscan, recuperan y leen publicaciones, es decir, son los usuarios de la información científica. Estos usuarios acceden diversas fuentes de información en línea, como bases de datos y bibliotecas digitales, y utilizan el medio electrónico también para comunicación informal, a partir de e-mails, redes sociales y foros (Pinheiro, 2012). Desde el punto de vista de los autores, el proceso de búsqueda de información científica en ambientes digitales es más rápido y muchas veces más eficiente. Sin embargo, todavía existen dificultades para muchos usuarios de la información científica en el uso de fuentes digitales relacionadas a la accesibilidad, dificultades de uso e infraestructura.

Los usuarios de sistemas digitales pueden ser clasificados en diferentes categorías, como expertos y principiantes a partir del uso del sistema y de los tipos de problemas que enfrentan en una interfaz (Cybis, Betiol, Faust, 2010). Los usuarios pueden ser identificados también de acuerdo con la frecuencia que utilizan el sistema en las categorías: primario, secundario y terciario. Los usuarios primarios son aquellos que potencialmente pueden ser convertidos en usuarios frecuentes del sistema, los secundarios utilizan el sistema ocasionalmente o por medio de un intermediario, por fin os terciarios son aquellos afectados por la introducción al sistema o influenciados a su compra (Rogers, Sharp, Preece, 2013).

Bohmerwald (2005) en referencia a investigaciones sobre sistemas de información y sus usuarios presenta un análisis más completo cuando utiliza características de estudios de usuarios y de estudios de usabilidad. La autora resalta que los profesionales involucrados con desarrollos de interfaces deben observar los usuarios, auxiliándose, por ejemplo, con los métodos de observación para prevenir problemas de usabilidad. Para Costa y Ramalho (2010), las investigaciones que se relacionan entre los estudios de usuarios y usabilidad son denominadas estudios híbridos de uso de la información. De acuerdo con las autoras tanto los estudios de 
usabilidad, como los estudios de usuarios tienen la preocupación de adecuar sistemas para satisfacer los usuarios, visando una mejor interacción.

\subsection{Estudios de Usabilidad en Repositorios}

A partir de búsquedas en bases de datos, fueron recuperados algunos estudios realizados sobre la usabilidad de repositórios institucionales. Y, pueden ser destacadas algunas investigaciones realizadas en el Brasil y en outros países.

Camargo y Vidotti (2008) evaluaron cualitativamente cinco repositorios digitales desarrollados en DSpace a partir de la observación de la interfaz. Los repositorios analizados fueron: RIDI; Diálogo Científico del Instituto Brasileño de Información en Ciencia y Tecnología (IBICT) (actualmente inexistente); ArXiv; SPARC; E-LIS y RepositoriUM. Los usuarios no fueron consultados, el estudio utilizó métodos de inspección. Las autoras constataron que los repositorios utilizan varios recursos de usabilidad, aunque algunos de ellos son usados de forma insatisfactoria con informaciones insuficientes sobre el repositorio, la institución, fechas de actualizaciones, trabajos en conjunto con otras instituciones, entrenamiento de usuarios e incentivo a la participación y utilización del repositorio.

Curty y Araújo (2008) evaluaron los criterios ergonómicos del RepositoriUM de la Universidad de Minho, en Portugal, que fue desarrollado con el software DSpace. El repositorio también fue observado a partir de un método de inspección. En este caso fue utilizada la lista de requisitos ergonómicos Ergolist. Las investigadores observaron que más de la mitad de los criterios de Ergolist no se aplicaban al repositorio. De las 90 cuestiones aplicables, 36\% recibieron parecer positivo.

En 2008, Tsakonas y Papatheodorou realizaron estudio sobre E-LIS, repositorio temático de e-prints en Ciencia de la Información. El software utilizado para este repositorio es el Eprint. El estudio enfocó las características de utilidad (usefulness) usabilidad (usability) y desempeño (performance) del repositorio, a partir de la perspectiva de sus usuarios. Fue utilizado un cuestionario en línea para la colecta de los datos. En este caso los autores afirmaron que el nivel de usabilidad del sistema para sus usuarios es alto por tratarse de un sistema de Acceso Abierto y que ofrece ciertas opciones de personalización.

En el estudio de Hyun e Yong de 2008 fue evaluado el repositorio común del Consorcio de repositorios digitales Sur-Coreanos (dCollection). Para este repositorio y todos los repositorios que hacen parte del consorcio fue desarrollado un software proprio, o dCollection system. Los autores concluyeron que sería necesario incluir el modelo conceptual de datos bibliográficos FRBR: Los requisitos funcionales de los registros bibliográficos (Functional Requirements for Bibliographic Records) en la catalogación del sistema, para facilitar la descripción de los metadatos y la recuperación de la información. Fue observado que los usuarios con poca experiencia tenían mayores expectativas en relación al sistema que los usuarios con más experiencia. La mayoría de los usuarios no estaban satisfechos con los colores y tenían dificultades en localizar los botones. 
En el estudio de Veiga, Machado y Alves de 2013, las autoras realizaron una evaluación de usabilidad del Repositorio Arca, que pertenece a la Fundación Oswaldo Cruz (Fiocruz) y que utiliza el software DSpace. El foco de la investigación fue el auto-archivamiento de los repositorios. El test fue realizado con seis (6) usuarios potenciales, que evaluaron el repositorio en tres módulos: diseño, terminología e información. Las autoras concluyeron que: aunque el diseño había sido el aspecto mejor evaluado por los usuarios, fueron detectadas necesidades de mejoría en las fuentes utilizadas, en la disposición y en los caracteres de los pantallazos. En relación a la terminología, deben ser corregidos términos ambiguos e inconsistentes. En relación a la información, los comandos e instrucciones no se mostraron claros.

En 2014 Silva realizó un estudio de usabilidad del Repositorio de la Universidad de Minho (RepositoriUM), consultando los diversos usuarios del repositorio. La autora aplicó un cuestionario para identificar el perfil de los usuarios y realizo un test de usabilidad con 16 participantes para identificar problemas en la interfaz del repositorio. De acuerdo con la autora, los usuarios tienen consciencia de que el repositorio concentra informaciones importantes y les gustaría utilizarlo con más frecuencia, pero tienen dificultad en encontrar las informaciones presentes en el mismo.

Las investigaciones elaboradas hasta entonces sobre usabilidad de repositorios traen consideraciones importantes en cuanto al desarrollo y mantenimiento de repositorios, y al respecto de los métodos aplicados en las investigaciones de usabilidad en repositorios. Sin embargo, se observa que es necesario un estudio más centrado en los usuarios, también en el contexto del Repositorio Institucional de la Universidad de Brasília.

\section{Metodología}

Para alcanzar los objetivos propuestos, fue desarrollado un estudio descriptivo, con abordajes cuantitativos y cualitativos. El método central en el desarrollo de la investigación fuees el estudio de caso. De acuerdo con Kafure et al (2007), es sugerida en la evaluación de la usabilidad la superposición de varios métodos y técnicas, para estar en condiciones de conocer mejor a los usuarios y definir mejor todos los aspectos de los sistemas. Es así, como en esta investigación para identificar el perfil de los usuarios, caracterizar la interfaz, identificar el modelo mental y las posibles dificultades de interacción en el proceso de recuperación de la información, fueron superpuestos y aplicados en la colecta y el análisis de los datos, los métodos y técnicas, a seguir:

- Cuestionarios: los cuestionarios en línea para identificar el perfil de los usuarios, los estudiantes de posgrado de la Universidad de Brasília (UnB).

- Evaluación heurística y grupo focal u objetivo: un método de inspección donde especialistas juzgan elementos de la interfaz según principios de usabilidad establecidos. Para caracterizar, en esta investigación, la interfaz 
según los principios de usabilidad establecidos. Fue una dinámica donde los participantes se sintieron cómodos y libres de hablar y comentar sus opiniones.

- Observación semi-participante, no participante y entrevista: para identificar el modelo mental de los usuarios.

- Análisis de la tarea y de la actividad, para obtención de la percepción de la tarea y su realización por parte de los usuarios. "Un principio fundamental de la ergonomía es el conocimiento de los usuarios y del trabajo a ser realizado" (Kafure et al, 2007). El trabajo es examinado de acuerdo a dos componentes básicos: la tarea y la actividad (Sebillote 1995; Guérin et al, 2001).

También fueron utilizadas las técnicas de levantamiento de datos como parte de la investigación. Las técnicas utilizadas para recolectar los dados de la investigación fueron: cuestionario; la evaluación heurística, un método de inspección donde especialistas juzgan elementos de la interfaz según principios de usabilidad establecidos; y la observación semi-participante con entrevista.

La identificación del perfil del usuario fue realizada a partir de la aplicación de cuestionarios en línea a los estudiantes de posgrado de la Universidad de Brasília (UnB). Los resultados fueron analizados a partir de estadística descriptiva. La muestra fue calculada a partir de los datos disponibles en el anuario estadístico más reciente de la UnB, de 2015, que presenta informaciones del período de 2010 a 2014. De acuerdo con este anuario, en 2014 habían 7.576 alumnos regulares registrados en cursos de posgrado Strictu Senso. Después de obtener los datos sobre la población, la muestra fue calculada de forma que el margen estándar fuese $5 \%$ y el nivel de confianza fuese $90 \%$. De acuerdo con Babbie (2003), la teoría de la probabilidad ofrece la siguiente fórmula para estimar la representatividad de muestras: $\mathrm{S}=$ tras: tatidonde $[\mathrm{P}]$ y $[\mathrm{Q}]$ son los parámetros poblacionales del binomial $(\mathrm{Q}=1-\mathrm{P}$ y $\mathrm{P}=1-\mathrm{Q})$, [n] es el número de casos y $[\mathrm{S}]$ es el error estándar. En el caso de esta investigación, se consideró el porcentaje como $50 \%$ para $\mathrm{P}$ y $\mathrm{Q}$, visando obtener mayor precisión en los cálculos de la muestra. A partir de los parámetros determinados, se obtuvo el resultado de que la muestra mínima necesaria para la población de estudiantes de posgrado de la UnB sería de 262 estudiantes. Fueran obtenidas 328 respuestas válidas, lo que sobrepasó la muestra mínima. El cuestionario fue divulgado por e-mail a los estudiantes de posgrado.

Para describir características de la interfaz del repositorio, fue realizada la evaluación heurística. En esta investigación, la evaluación fue elaborada a partir de la combinación de diferentes modelos y sugerencias presentes en la literatura. Son ellas: las diez heurísticas de Nielsen (1995), de las recomendaciones de Dias (2006) sobre requisitos de usabilidad para evaluación heurística y del modelo de plantilla de Rocha (2014), la autora que realizó una evaluación heurística de revistas en línea y describió los problemas encontrados en cuadros.

En esta investigación fue elaborado un El formato fue elaborado de una forma tal que quedase más adecuado al contexto del RIUnB. Hubo 12 evaluadores participantes, que eran alumnos de pregrado en Bibliotecología y Archivología de 
la Facultad de Ciencia de la Información de la Universidad de Brasília. Los evaluadores recibieron créditos académicos por la participación en la investigación. Además de las evaluaciones realizadas individualmente conforme los criterios ergonómicos establecidos, los evaluadores participaron de grupos focales y describieron sus impresiones sobre la interfaz del repositorio en informes.

La identificación del modelo mental de los usuarios fue realizada a partir de la observación semi-participante, que combina características de la observación noparticipante con la observación-participante. La primera permite que los usuarios se expresen libremente, la segunda permite que el investigador haga entrevista con los usuarios (Flick, 2010). En este estudio, fue observada la interacción entre los usuarios de posgrado y la interfaz del repositorio durante la busca por un documento. Los estudiantes de posgrado interesados en participar entraron en contacto por e-mail, informando la disponibilidad para realizar un encuentro. Fueron entrevistados 12 usuarios, conforme las recomendaciones para estudios de usabilidad de Alroobaea y Mayhew (2014). Los participantes tuvieron libertad para escoger el local de la entrevista y el computador para consultar el repositorio. La entrevista fue conducida de la siguiente forma: Primero, fueron hechas preguntas sobre la percepción del usuario sobre el Repositorio, que sirven para la identificación de la Tarea. Después de estas preguntas, los participantes eran convidados a realizar una Tarea en la utilización del repositorio, mientras eran observadas sus interacciones y hechas anotaciones en papel, sobre las mismas. La tarea consistió en uso de la interfaz del repositorio para la lectura de informaciones y búsqueda de documentos disponibles. Después del uso del repositorio, fueron realizadas otras preguntas a los usuarios relacionadas a la Tarea, y de esta forma, pudo ser comparada la percepción de la Tarea y la realización de la misma (Actividad) por parte de los usuarios. Posteriormente, fue realizado un análisis sobre el modelo mental. De esta manera se aplicó la entrevista y su análisis de acuerdo con el abordaje metodológico de Kafure, en el que son comparadas la Tarea y la Actividad, para identificar las dificultades y direccionar el aumento de la usabilidad de los instrumentos tecnológicos, de acuerdo con las necesidades y expectativas del usuario (Kafure, 2004).

Las dificultades de interacción fueron observadas a partir de los métodos anteriormente descritos. En el cuestionario fue incluida una pregunta opcional sobre dificultades de uso. Durante la evaluación heurística, los evaluadores apuntaron problemas de usabilidad que pueden traer dificultades a los usuarios. Y durante la observación semi-participante, fueron descritas y comentadas dificultades observadas durante la interacción.

Resumidamente, los métodos, técnicas de colecta y de análisis del estudio son descritos en el cuadro 1, según los objetivos específicos: 
Cuadro 1 - Métodos, técnicas e instrumentos del estudio

\begin{tabular}{|l|l|l|l|l|}
\hline Objetivos & Métodos & $\begin{array}{l}\text { Recolección de } \\
\text { datos }\end{array}$ & $\begin{array}{l}\text { Instrumentos de } \\
\text { recolección }\end{array}$ & $\begin{array}{l}\text { Técnicas de } \\
\text { análisis }\end{array}$ \\
\hline OBJ 1(perfil) & $\begin{array}{l}\text { Estudio de caso e } \\
\text { levantamiento }\end{array}$ & Cuestionario & $\begin{array}{l}\text { Web-survey } \\
\text { (Google } \\
\text { Formularios) }\end{array}$ & $\begin{array}{l}\text { Estadística } \\
\text { descriptiva }\end{array}$ \\
\hline OBJ 2 (interfaz) & $\begin{array}{l}\text { Estudio de caso e } \\
\text { levantamento }\end{array}$ & $\begin{array}{l}\text { Evaluación } \\
\text { Heurística }\end{array}$ & $\begin{array}{l}\text { Lista de } \\
\text { heurísticas de } \\
\text { usabilidade. } \\
\text { Guion de grupo } \\
\text { focal }\end{array}$ & $\begin{array}{l}\text { Categorización e } \\
\text { interpretación de } \\
\text { datos. Estadística } \\
\text { descriptiva }\end{array}$ \\
\hline $\begin{array}{l}\text { OBJ 3 (modelo } \\
\text { mental) }\end{array}$ & Estudio de caso & $\begin{array}{l}\text { Observación } \\
\text { semi-participante } \\
\text { con entrevista }\end{array}$ & $\begin{array}{l}\text { Guion de } \\
\text { entrevista y de } \\
\text { observación }\end{array}$ & $\begin{array}{l}\text { Categorización e } \\
\text { interpretación de } \\
\text { datos }\end{array}$ \\
\hline $\begin{array}{l}\text { OBJ 4 } \\
\text { (dificultades) }\end{array}$ & $\begin{array}{l}\text { Estudio de caso } \\
\text { e levantamiento }\end{array}$ & $\begin{array}{l}\text { Observación } \\
\text { semi-participante } \\
\text { con entrevista, } \\
\text { evaluación } \\
\text { heurística y } \\
\text { cuestionario }\end{array}$ & $\begin{array}{l}\text { Guion de } \\
\text { entrevista y de } \\
\text { observación. } \\
\text { Lista de } \\
\text { heurísticas de } \\
\text { usabilidad. } \\
\text { Web-survey }\end{array}$ & $\begin{array}{l}\text { Categorización e } \\
\text { interpretación de } \\
\text { datos. Estadística } \\
\text { descriptiva }\end{array}$ \\
\hline
\end{tabular}

\section{Resultados}

Los resultados, a continuación, son presentados de acuerdo con los objetivos específicos de la investigación.

\subsection{Identificación del Perfil de los Usuarios}

El perfil de los usuarios del RIUnB, que participaron en esta investigación, es el de estudiantes de posgrado de maestría y doctorado de diversas áreas del conocimiento, principalmente, de las áreas de Ciencias Humanas y Ciencias Sociales Aplicadas. Los principales temas de investigación se refieren a la Educación, Historia, Salud, Gestión Pública y Letras. En relación a la edad, 60\% de los usuarios poseen entre 25 y 35 años de edad. En esta investigación fue posible obtener una muestra variada, con participantes de diferentes cursos y áreas del conocimiento.

\subsection{Los usuarios indicaron utilizar diferentes fuentes de información.}

Conforme descrito por Pinheiro (2007), los usuarios académicos utilizan fuentes de información en línea formales e informales para sus necesidades de información científica. En esta investigación, los usuarios indicaron la utilización de la Internet en gran escala en las investigaciones académicas, principalmente en Google y Google Académico. Algunos usuarios indicaron utilizar fuentes informales como las redes sociales. Este hecho demuestra la importancia de los servicios de búsqueda para la visibilidad del repositorio y también para la recuperación de las informaciones en el 
repositorio. Sin embargo, durante las observaciones, algunos usuarios se mostraron sorprendidos al descubrir aspectos de la producción científica realizada dentro de su propia Universidad. A partir del uso de la interfaz del repositorio, los usuarios observaron trabajos de interés producidos y dirigidos por sus profesores y tutores, así como los trabajos de otros investigadores cercanos que desconocían. Este hecho demuestra que aunque es interesante recuperar las informaciones del repositorio por buscadores, la interfaz del sistema posibilita la recuperación de informaciones sobre la producción científica en la UnB. Todavía existe la necesidad de una reflexión de parte de los usuarios al respecto de este potencial del repositorio, porque este hecho no fue comentado durante la realización de la tarea y por lo tanto, no hacia parte del modelo mental de los usuarios.

El perfil de los usuarios del RIUnB, que participaron en esta investigación, es el de estudiantes de posgrado de maestría y doctorado de diversas áreas del conocimiento, principalmente, de las áreas de Ciencias Humanas y Ciencias Sociales Aplicadas. Los principales temas de investigación se refieren a la Educación, Historia, Salud, Gestión Pública y Letras. En relación a la edad, $60 \%$ de los usuarios poseen entre 25 y 35 años de edad. En esta investigación fue posible obtener una muestra variada, con participantes de diferentes cursos y áreas del conocimiento.

Los usuarios indicaron utilizar diferentes fuentes de información. Conforme descrito por Pinheiro (2007), los usuarios académicos utilizan fuentes de información en línea formales e informales para sus necesidades de información científica. En esta investigación, los usuarios indicaron la utilización de la Internet en gran escala en las investigaciones académicas, principalmente en Google y Google Académico. Algunos usuarios indicaron utilizar fuentes informales como las redes sociales. Este hecho demuestra la importancia de los servicios de búsqueda para la visibilidad del repositorio y también para la recuperación de las informaciones en el repositorio. Sin embargo, durante las observaciones, algunos usuarios se mostraron sorprendidos al descubrir aspectos de la producción científica realizada dentro de su propia Universidad. A partir del uso de la interfaz del repositorio, los usuarios observaron trabajos de interés producidos y dirigidos por sus profesores y tutores, así como los trabajos de otros investigadores cercanos que desconocían. Este hecho demuestra que aunque es interesante recuperar las informaciones del repositorio por buscadores, la interfaz del sistema posibilita la recuperación de informaciones sobre la producción científica en la UnB. Todavía existe la necesidad de una reflexión de parte de los usuarios al respecto de este potencial del repositorio, porque este hecho no fue comentado durante la realización de la tarea y por lo tanto, no hacia parte del modelo mental de los usuarios.

Sin embargo, los usuarios poco utilizan repositorios para hacer búsquedas académicas. Cuando usan un repositorio, generalmente consultan el de su propia institución, en este caso el de UnB. También, son consultados otros repositorios, como el de la Universidad de San Paulo (USP) y el de la Universidad Estatal de Campinas (Unicamp). En el caso del RIUnB, se considera que la mayor parte de los usuarios son principiantes, debido a la baja frecuencia de uso. Entre los participantes del cuestionario, apenas 53 (16\%) utilizan el repositorio en frecuencia mensual o semanal. Se resalta también que casi un tercio de los participantes (33\%) 
no tenían conocimiento sobre la existencia del repositorio. Este hecho indica que los usuarios utilizan poco el repositorio, como también tienen poco conocimiento a su respecto. Sería interesante impulsar prácticas de divulgación sobre el RIUnB entre los estudiantes de la UnB. El entrenamiento de bases de datos se presenta como una buena perspectiva para difundir el uso del repositorio y facilitar la interacción entre este y los usuarios, en la manifestación positiva de los participantes del entrenamiento, al afirmar que no tienen grandes dificultades de interacción y al mostrar conocimiento sobre el funcionamiento del repositorio.

Cuando consultan el repositorio, los estudiantes tienen interés, principalmente, en las búsquedas por un documento específico, indicando que consultan el repositorio al saber de la existencia de un documento. Sin embargo, la búsqueda por autor y asunto también son de interés a los usuarios, lo que hace necesario mejorar el sistema de búsqueda y recuperación de la información.

En el Gráfico 1, los participantes indican los motivos que los llevan o los llevarían a utilizar el RIUnB. Las respuestas fueran hechas con base en una escala de 1 a 5, donde 1 - improbable y 5 - muy probable. Los posibles motivos de uso son: encontrar un documento específico, encontrar literatura científica sobre un asunto específico, encontrar literatura científica de un autor específico, encontrar documentos científicos de autoría propia, observar informaciones sobre la producción científica de la UnB, mirar estadísticas de acceso y descarga de documentos. 
Gráfico 1 - Motivos de uso del RIUnB (n=328)

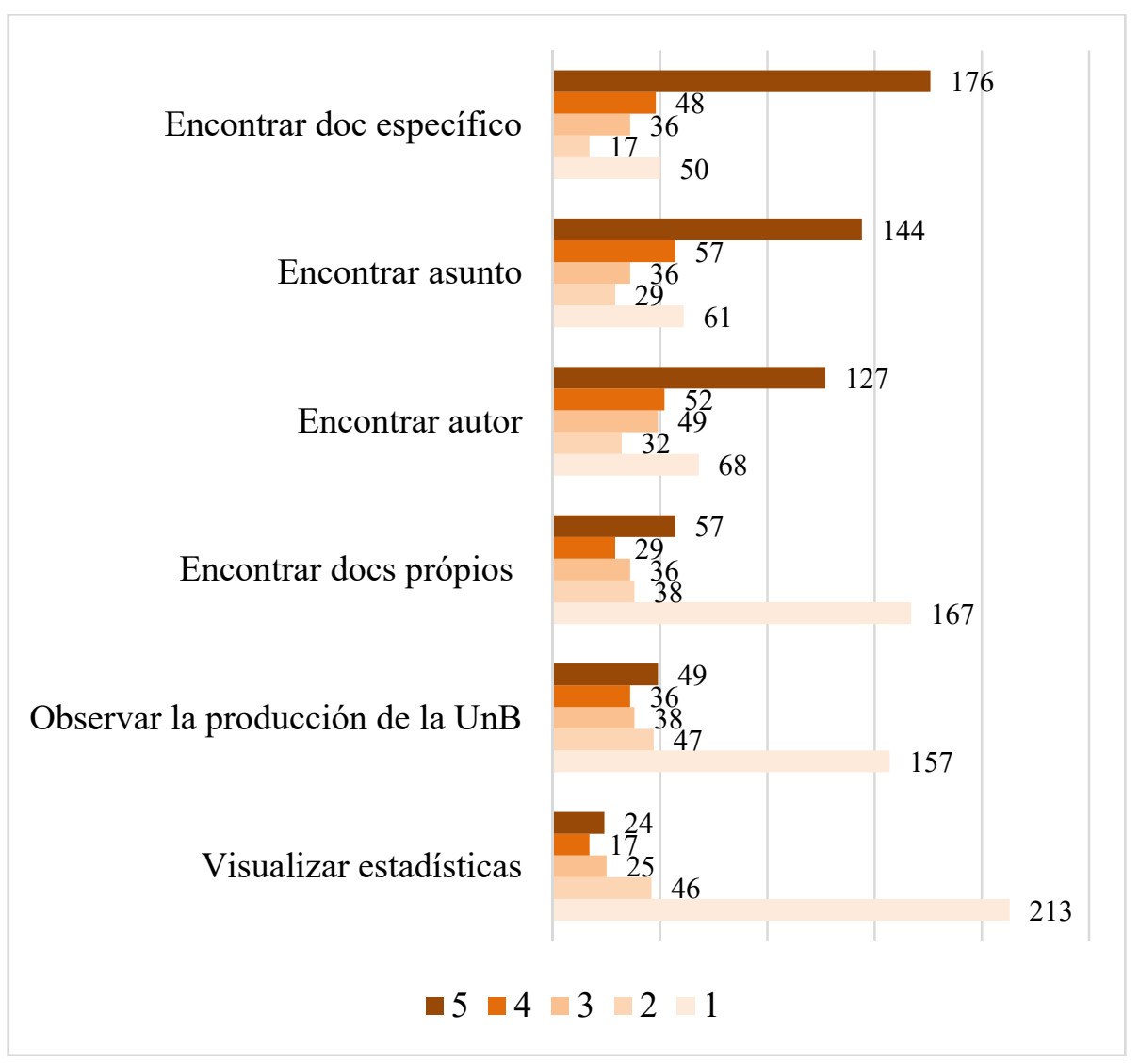

Fuente: Elaboración propia.

\subsection{Evaluación de la usabilidad de la interfaz del RIUnB}

En el Cuadro 1, son presentados algunos resultados de la evaluación heurística. Están organizados de acuerdo a la numeración de cada heurística, los criterios y de algunos ejemplos de problemas, de dificultades y de las recomendaciones encontradas.

Cuadro 2 - Algunos resultados de la evaluación heurística

\begin{tabular}{|c|l|l|l|l|}
\hline \multicolumn{1}{|c|}{ Heurística } & \multicolumn{1}{c|}{ Criterio } & $\begin{array}{l}\text { Número de Problemas } \\
(\mathrm{NP})\end{array}$ & \multicolumn{1}{|c|}{$\begin{array}{c}\text { Algunas dificultades } \\
\text { Algunas } \\
\text { recomendaciones }\end{array}$} \\
\hline $\begin{array}{l}\text { 1 - Visibilidad del } \\
\text { estado del sistema. }\end{array}$ & $\begin{array}{l}\text { Mantener los } \\
\text { usuários } \\
\text { informados, por } \\
\text { médio de una } \\
\text { retroalimentación } \\
\text { adecuada. }\end{array}$ & $\begin{array}{l}\mathrm{NP}=18, \text { por ejemplo, } \\
\text { ausencia explicaciones } \\
\text { relacionadas al } \\
\text { almacenamiento de } \\
\text { documentos, al sistema } \\
\text { de búsqueda y a la } \\
\text { política del sitio web. }\end{array}$ & $\begin{array}{l}\text { Desconocimiento en } \\
\text { relación a la tarea ser } \\
\text { realizada y a sus } \\
\text { etapas. }\end{array}$ & $\begin{array}{l}\text { Inserir explicaciones } \\
\text { sobre los } \\
\text { documentos, los } \\
\text { tipos de búsqueda y } \\
\text { la política del } \\
\text { repositorio. }\end{array}$ \\
\end{tabular}




\begin{tabular}{|c|c|c|c|c|}
\hline Heurística & Criterio & $\begin{array}{c}\text { Número de Problemas } \\
\text { (NP) }\end{array}$ & Algunas dificultades & $\begin{array}{c}\text { Algunas } \\
\text { recomendaciones }\end{array}$ \\
\hline $\begin{array}{l}2 \text { - Adecuación } \\
\text { entre el sistema y el } \\
\text { mundo real. }\end{array}$ & $\begin{array}{l}\text { Utilizar un } \\
\text { lenguaje familiar } \\
\text { al usuario. }\end{array}$ & $\begin{array}{l}\mathrm{NP}=8, \text { por ejemplo, } \\
\text { traducción incompleta } \\
\text { del sitio web en otros } \\
\text { idiomas. }\end{array}$ & $\begin{array}{l}\text { Incomprensión del } \\
\text { idioma utilizado en el } \\
\text { repositorio. }\end{array}$ & $\begin{array}{l}\text { Traducir las páginas } \\
\text { del sitio web de } \\
\text { acuerdo a los } \\
\text { idiomas escogidos } \\
\text { por el usuario. }\end{array}$ \\
\hline $\begin{array}{l}3 \text { - Control y } \\
\text { libertad del usuario. }\end{array}$ & $\begin{array}{l}\text { Facilitar las } \\
\text { acciones de hacer } \\
\text { y rehacer cuando } \\
\text { el usuario se } \\
\text { equivoca. }\end{array}$ & $\begin{array}{l}\mathrm{NP}=16 \text {, por ejemplo, } \\
\text { ausencia de opción } \\
\text { para retornar a la } \\
\text { página anterior } \\
\text { rápidamente. Ausencia } \\
\text { del cuadro de búsqueda } \\
\text { en diversas páginas del } \\
\text { RIUnB. }\end{array}$ & $\begin{array}{l}\text { Los usuarios pueden } \\
\text { tener dificultades en } \\
\text { recomenzar sus } \\
\text { acciones después de } \\
\text { equivocarse. }\end{array}$ & $\begin{array}{l}\text { Inserir opciones para } \\
\text { retornar a la página } \\
\text { sin perder el } \\
\text { progreso de la } \\
\text { búsqueda. Inserir un } \\
\text { cuadro de búsqueda } \\
\text { en todas las páginas. }\end{array}$ \\
\hline $\begin{array}{l}4 \text { - Consistencia y } \\
\text { normas. }\end{array}$ & $\begin{array}{l}\text { Padronización de } \\
\text { las páginas del } \\
\text { repositorio. }\end{array}$ & $\begin{array}{l}\mathrm{NP}=21 \text {, por ejemplo, } \\
\text { ausencia de } \\
\text { estandarización de las } \\
\text { fuentes y de las fechas } \\
\text { de publicación de los } \\
\text { ítems. No hay } \\
\text { distinción entre los } \\
\text { colores de los } \\
\text { elementos a los que se } \\
\text { puede hacer clic y los } \\
\text { que no. }\end{array}$ & $\begin{array}{l}\text { La estética del } \\
\text { repositorio } \\
\text { compromete el acceso } \\
\text { a los textos. }\end{array}$ & $\begin{array}{l}\text { Elegir un estándar } \\
\text { tipográfico y para } \\
\text { representar fechas. } \\
\text { Establecer } \\
\text { diferencias entre los } \\
\text { elementos a los que } \\
\text { se les puede o no } \\
\text { hacer clic. }\end{array}$ \\
\hline $\begin{array}{l}5 \text { - Prevención de } \\
\text { erros. }\end{array}$ & $\begin{array}{l}\text { Eliminar } \\
\text { condiciones } \\
\text { favorables al } \\
\text { error. }\end{array}$ & $\begin{array}{l}\mathrm{NP}=8, \text { por ejemplo, } \\
\text { ausencia sugerencias de } \\
\text { búsqueda. Los } \\
\text { resultados recuperados } \\
\text { ni siempre están } \\
\text { relacionados a los } \\
\text { términos utilizados en } \\
\text { la búsqueda. }\end{array}$ & $\begin{array}{l}\text { Dificultad en la } \\
\text { recuperación de la } \\
\text { información en los } \\
\text { procesos de búsqueda } \\
\text { del repositorio. }\end{array}$ & $\begin{array}{l}\text { Actualizar el sistema } \\
\text { de búsqueda y } \\
\text { trabajar la } \\
\text { descripción de los } \\
\text { documentos según el } \\
\text { modelo FRBR. }\end{array}$ \\
\hline $\begin{array}{l}6 \text { Reconocimiento } \\
\text { en vez de } \\
\text { memorización. }\end{array}$ & $\begin{array}{l}\text { El usuario debe } \\
\text { comprender y } \\
\text { recordar } \\
\text { instrucciones de } \\
\text { la interfaz } \\
\text { fácilmente. }\end{array}$ & $\begin{array}{l}\mathrm{NP}=2, \text { por ejemplo, } \\
\text { ausencia de } \\
\text { recomendaciones para } \\
\text { hacer búsquedas. No es } \\
\text { claro el significado de } \\
\text { números al lado de las } \\
\text { comunidades de } \\
\text { documentos. }\end{array}$ & $\begin{array}{l}\text { Los usuarios ni } \\
\text { siempre comprenden o } \\
\text { se acuerdan de algunas } \\
\text { etapas del proceso de } \\
\text { búsqueda. }\end{array}$ & $\begin{array}{l}\text { Inserir indicaciones } \\
\text { para la búsqueda y } \\
\text { explicar los } \\
\text { significados de los } \\
\text { números. }\end{array}$ \\
\hline $\begin{array}{l}7 \text { - Flexibilidad y } \\
\text { eficiencia de uso. }\end{array}$ & $\begin{array}{l}\text { Atajos pueden } \\
\text { acelerar la } \\
\text { interacción } \\
\text { cuando se trate de } \\
\text { usuarios con } \\
\text { experiencia. }\end{array}$ & $\begin{array}{l}\mathrm{N}=8, \text { por ejemplo, } \\
\text { demora en la } \\
\text { recuperación de la } \\
\text { información, para } \\
\text { usuarios con } \\
\text { experiencia en la } \\
\text { interacción con la } \\
\text { interfaz. }\end{array}$ & $\begin{array}{l}\text { Algunos procesos se } \\
\text { hacen más largos, sin } \\
\text { necesidad. }\end{array}$ & $\begin{array}{l}\text { Inserir un enlace a la } \\
\text { página principal. } \\
\text { Exhibir los } \\
\text { documentos al } \\
\text { terminar la } \\
\text { búsqueda. Incluir } \\
\text { acciones que } \\
\text { favorezcan } \\
\text { diferentes tipos de } \\
\text { usuarios. }\end{array}$ \\
\hline $\begin{array}{l}8 \text { - Estética y } \\
\text { diseño minimalista. }\end{array}$ & $\begin{array}{l}\text { Evitar } \\
\text { información } \\
\text { irrelevante o que } \\
\text { es raramente } \\
\text { necesaria. Los } \\
\text { textos deben estar } \\
\text { claros y concisos. }\end{array}$ & $\begin{array}{l}\mathrm{N}=12 \text {, por ejemplo, el } \\
\text { eslogan tiene un } \\
\text { tamaño pequeño y de } \\
\text { difícil lectura. }\end{array}$ & $\begin{array}{l}\text { Dificultad de } \\
\text { visualización del } \\
\text { eslogan. }\end{array}$ & $\begin{array}{l}\text { En general, el RIUnB } \\
\text { presenta una interface } \\
\text { sin demasiado } \\
\text { contenido, pero } \\
\text { algunos elementos de } \\
\text { los textos pueden ser } \\
\text { mejor formateados } \\
\text { para facilitar la } \\
\text { lectura. }\end{array}$ \\
\hline
\end{tabular}




\begin{tabular}{|c|c|c|c|c|}
\hline Heurística & Criterio & $\begin{array}{l}\text { Número de Problemas } \\
\text { (NP) }\end{array}$ & Algunas dificultades & $\begin{array}{c}\text { Algunas } \\
\text { recomendaciones }\end{array}$ \\
\hline $\begin{array}{l}9 \text { - Ayuda para los } \\
\text { usuarios reconocer, } \\
\text { diagnosticar y } \\
\text { recuperarse de los } \\
\text { errores. }\end{array}$ & $\begin{array}{l}\text { Caso haya } \\
\text { problemas, las } \\
\text { ayudas y suporte } \\
\text { deben ser claras. }\end{array}$ & $\begin{array}{l}\mathrm{NP}=6 \text {, por ejemplo, } \\
\text { hay mensajes de error } \\
\text { en idioma diferente al } \\
\text { escogido por el usuario. } \\
\text { Ausencia de un } \\
\text { mensaje indicando que } \\
\text { no hay resultados } \\
\text { cuando una búsqueda } \\
\text { no recupera } \\
\text { documentos. }\end{array}$ & $\begin{array}{l}\text { El usuario no recibe } \\
\text { retroalimentación } \\
\text { adecuada del sistema. } \\
\text { Los textos en idiomas } \\
\text { diferentes al del } \\
\text { usuario pueden ser } \\
\text { incomprendidos. }\end{array}$ & $\begin{array}{l}\text { Traducir mensajes } \\
\text { de error e inserir } \\
\text { mensajes para las } \\
\text { búsquedas sin } \\
\text { resultados. }\end{array}$ \\
\hline $\begin{array}{l}10 \text { - Ayuda y } \\
\text { documentación. }\end{array}$ & $\begin{array}{l}\text { Puede ser } \\
\text { necesario ofrecer } \\
\text { ayuda y textos de } \\
\text { apoyo. }\end{array}$ & $\begin{array}{l}\mathrm{NP}=7, \text { por ejemplo, } \\
\text { no es posible visualizar } \\
\text { imágenes de los } \\
\text { manuales y algunas } \\
\text { informaciones están } \\
\text { incompletas. }\end{array}$ & $\begin{array}{l}\text { El usuario que necesita } \\
\text { consultar los } \\
\text { repositorios puede no } \\
\text { encontrar la } \\
\text { instrucción que busca. }\end{array}$ & $\begin{array}{l}\text { Completar los } \\
\text { manuales de ayuda a } \\
\text { los usuarios. }\end{array}$ \\
\hline
\end{tabular}

Fuente: Elaboración propia

\subsubsection{Consideraciones de la Evaluación Heurística}

En esta investigación fue posible constatar que en general los usuarios esperan un uso fácil de la interfaz y encontrar informaciones que estén de acuerdo a sus necesidades de búsqueda en el RIUnB. Sin embargo, fueron detectadas dificultades durante el uso de la interfaz y en la recuperación de la información, que no permitieron satisfacer sus necesidades de información. Fueran identificados muchos problemas en la interfaz del RIUnB a partir de la evaluación heurística. Destacase como problemas severos la falta de precisión en el sistema de búsqueda del repositorio y falta de informaciones sobre política, depósito de documentos y funcionamiento del RIUnB.

Los evaluadores, durante las discusiones, destacaran el diseño antiguo del repositorio, y la falta de actualización del contenido de las redes sociales como factores que influencian su percepción a respecto del uso del repositorio. A partir de las observaciones de uso por los usuarios fue posible identificar los mismos problemas indicados por los evaluadores. Las dificultades observadas durante la interacción son provenientes de las discrepancias entre el modelo mental de los usuarios y el modelo conceptual de los diseñadores, lo que lleva a los usuarios a proporcionar diversos engaños durante la interacción.

Acerca de la recuperación de la información, los usuarios respondieron en el cuestionario que utilizan o utilizarían el RIUnB principalmente para encontrar documentos específicos, realizar búsquedas por asunto y para encontrar documentos de un autor específico. Sin embargo, en la evaluación heurística fue constada la dificultad de la recuperación de la información por asunto, pues los resultados de la búsqueda ni siempre coinciden con los términos utilizados. También durante las observaciones, los usuarios tuvieron dificultad en encontrar documentos realizando búsquedas por autor. Este hecho ocurrió debido a que el sistema no recupera nombre de autores de manera directa, es decir, por Nombre seguido del Apellido. Estos problemas detectados pueden ser graves, pues afectan 
las principales formas de recuperación de la información utilizadas por los usuarios. La recuperación de la información eficiente posibilita la satisfacción de los usuarios en relación a sus necesidades informacionales. Por lo tanto, los problemas alcanzan la satisfacción de los usuarios y la usabilidad del sistema.

También fueron detectadas dificultades en la recuperación de la información que estaban relacionadas a la falta de contenido del repositorio. Aunque el RIUnB sea reconocido por su cantidad de documentos inseridos en el sistema, los usuarios no encontraron algunos documentos más antiguos o recientes en el repositorio.

Los evaluadores apuntaran muchos puntos positivos del repositorio, como las estadísticas de acceso, que pueden servir como un incentivo de uso del RIUnB. El lenguaje utilizado en los textos del repositorio en general fue considerada adecuada a los usuarios. La posibilidad de visualizar metadatos antes de descargar un archivo, así como la permisión para acceder los documentos sin la necesidad de registrarse en el sistema son puntos positivos en la interacción con el RIUnB. Las observaciones indican que a pesar de las dificultades, después del uso del repositorio, los usuarios aprendieron ciertos aspectos sobre la interfaz, como la posibilidad de observar la producción académica de los investigadores y profesores conocidos, por eso muchos quedaron satisfechos con el uso de la interfaz.

Como sugerencia, los evaluadores apuntan que sería interesante la inclusión de funcionalidades sociales en el RIUnB, que permitirían la interacción directa con autores de documentos. En los datos del cuestionario, algunos usuarios indicaran usar redes sociales académicas que tienen estas funcionalidades.

\subsection{Identificación del Modelo Mental de los Usuarios}

A partir de las observaciones de uso por los usuarios fue posible identificar como la interfaz del RIUnB afecta el modelo mental de los usuarios, como también fue posible recolectar datos al respecto de las percepciones de los usuarios sobre el RIUnB.

Los usuarios que quedaron insatisfechos después de la interacción con el repositorio tuvieron dificultades en la recuperación de la información debido a la falta de contenido o problemas con el sistema de búsqueda del repositorio.

Aunque los estudiantes de posgrado utilicen mucho la Internet para realizar investigaciones, estos acceden poco al RIUnB para investigaciones académicas. Estos usuarios acceden principalmente al Google y Google Académico para realizar investigaciones. El Google también es la forma principal de acceso al repositorio. Durante las observaciones se constató que muchos participantes buscan el repositorio en Google para acceder a su interfaz.

\subsection{Identificación de dificultades en el uso}

Al final del cuestionario, los participantes respondieran a una cuestión abierta, opcional a respecto de dificultades de uso del RIUnB. Muchas declaraciones de dificultades se refieren al sistema de búsqueda y a la presentación de los resultados de búsqueda del repositorio. Un total de 29 usuarios declararan tener este tipo de dificultad. Ocho usuarios afirmaran que generalmente tienen problemas con la 
colección de documentos del repositorio, comentando que a veces hay demora para depósito de documentos más nuevos, o no hay teses y disertaciones más antiguas. Cinco usuarios afirmaran que tienen problemas con la operación del sistema, pues muchas veces no es posible conectarse al repositorio. Tres participantes del estudio afirmaran que debería haber más divulgación del Repositorio.

\section{Conclusiones}

El objetivo general de la investigación, que fue describir la interacción de los usuarios estudiantes de posgrados con el RIUnB fue alcanzado. Para llegar a este objetivo, fue necesario conocer los usuarios, caracterizar la interfaz del Repositorio y observar su uso. Después del proceso de investigación, es posible decir que la interacción entre los usuarios y el repositorio ocurre muchas veces de forma satisfactoria, pero también existen dificultades que afectan la satisfacción de los usuarios en el proceso de interacción. Todavía existen expectativas y necesidades de información de los usuarios que precisarían ser atendidas. Por eso, es necesario mejorar diversos aspectos del repositorio, apuntados en los resultados de esta investigación. Aunque los usuarios utilicen poco el repositorio, reconocen su potencial para investigación y recuperación de la información académica, de esa manera, sugieren mejorías como adicionar más contenido o divulgar más el servicio.

Este estudio investigó la usabilidad a partir de la perspectiva de sus usuarios estudiantes de posgrado de la UnB. Sin embargo, una observación completa de la usabilidad del repositorio contemplaría también un estudio a partir de la perspectiva del equipo gestor del repositorio. Por lo tanto, un estudio futuro relevante sería analizar la usabilidad del Repositorio de la UnB a partir de la perspectiva de los bibliotecarios, analistas de sistemas y otros profesionales responsables por el desarrollo y gestión del servicio. Otro aspecto pertinente que no fue contemplado en este estudio se refiere a la accesibilidad del repositorio. Se espera que los repositorios institucionales, incluyendo el RIUnB, atiendan a toda la comunidad científica, inclusive personas con diferentes deficiencias, en un diseño universal, para acceder y usar el repositorio con facilidad, para el mayor número de personas posible, sin la necesidad de adaptarlos o rediseñarlos de una forma especial.

Algunos estudios de usabilidad sobre diferentes repositorios habían sido realizados antes de esta investigación. Sin embargo, puede destacarse la contribución de esta investigación por la realización de un estudio de usabilidad con los usuarios del repositorio. De esta manera, los datos obtenidos fueron compatibles con la realidad de los usuarios y de los usuarios potenciales del repositorio. A partir de la consulta con los usuarios, fue posible realizar una evaluación de la usabilidad del repositorio centrada en los usuarios y en los aspectos humanos de la comunicación científica.

Finalmente, se sabe que la comunicación de investigaciones científicas es esencial para el desarrollo de la Ciencia y actualmente, el movimiento del Acceso Abierto y los repositorios digitales tienen un papel importante de mediación en la comunicación y en la recuperación de la información científica. Los resultados de 
esta investigación traen informaciones relevantes para la mejoría de un repositorio digital de acceso abierto y para la mejoría de la recuperación de la información por sus usuarios. Por lo tanto, se espera que esta investigación haya contribuido con el aumento de la usabilidad en el desarrollo de la interfaz del RIUnB, como de otros repositorios digitales que puedan disponer de las recomendaciones apuntadas en este trabajo.

\section{Referencias bibliográficas}

Alroobaea R.; Mayhew, Pam J. (2014) How many participants are really enough for usability studies? In: Science and Information Conference. Londres.

Associação Brasileira De Normas Técnicas (2011) ISO 9241-11. Requisitos ergonômicos para o trabalho com dispositivos de interação visual. Parte 11: orientações sobre usabilidade. Rio de Janeiro, ABNT.

Babbie, E. (2003) Métodos de pesquisa de Survey. Belo Horizonte, UFMG.

Bégault, B. (2009). O periódico científico, um papel para a mediação de informação entre pesquisadores: qual seu futuro no ambiente digital? RECIIS - R. Eletr. de Com. Inf. Inov. Saúde. Rio de Janeiro, 3 (3), 91-96.

Bjork, B. C. (2007) A model of scientific communication as a global distributed information system. <http://www.informationr.net/ir/12-2/paper307.html> [Consulta: 24/01/2015]

Bohmerwald, P. (2005) Uma proposta metodológica para avaliação de bibliotecas digitais: usabilidade e comportamento de busca por informação na Biblioteca Digital da PUCMinas. Ciência da Informação, 1 (34)

Camargo, L. S. A. Vidotti, S. B. G. (2008) Uma estratégia de avaliação de repositórios digitais. Seminário internacional de Bibliotecas Universitárias, XV. São Paulo, Brasil.

Costa, L. F. da; Ramalho, F. A. (2010) A usabilidade nos estudos de uso da informação: em cena usuários e sistemas interativos de informação. Perspectivas em ciência da informação, 1 (15).

Costa, M. P. da. (2014) Características e contribuições da Via Verde para o acesso aberto à informação científica na América Latina. Brasília, Universidade de Brasília.

Curty, R. G.; Araújo, N. C. de. (2008) Análise da usabilidade de interface de repositório institucional: enfoque nos princípios ergonômicos. Encontro Nacional da Associação Nacional de Pesquisa em Ciência da Informação, XX, São Paulo, Brasil.

Cybis, W.; Betiol, A. H.; Faust, R.. (2010) Ergonomia e usabilidade: conhecimentos, métodos e aplicações. São Paulo, Novatec.

Dias, C. (2006) Usabilidade na web: criando portais mais acessiveis. Rio de Janeiro, Alta Books.

Dix, A.; Finlay, J.; Abowd, G. D.; Beale, R. (2004) Human-Computer Interaction. Edinburgh, Pearson.

Egert, A.; Gershon, N. D.; Van Der Veer, G. C. (2012) Human-Computer Interaction: introduction and overview. Kunstl Intell, 2 (26), 121-126.

Farias, R. A. N.; Galindo, Marcos. (2016) Análise de comportamento do uso de repositórios digitais de universidades federais brasileiras. Páginas $A \&$ B. Arquivos \& Bibliotecas, v. 6, 3-18.

Flick, U. (2010) An introduction to qualitative research. London, SAGE. 
Freitas, M. A. de; Silva, P. N. da; Guimarães, J. de F. (2009) Repositórios institucionais: a experiência da Universidade de Brasília, en Implantação e gestão de repositórios institucionais: politicas, memórias, livre acesso e preservação. Salvador: EDUFBA.

Guérin, F.; Laville, A.; Daniellou, F.; Duraffourg, J.; Kerguelen, A. (2001) Compreender o trabalho para transformá-lo: a prática da ergnomia. São Paulo: Editora Edgar Blücher Ltda.

Harnad, Stevan; Brody, T. (2004) Comparing the Impact of Open Access (OA) vs. Non-OA Articles in the Same Journals. D-Lib Magazine, 6 (10).

Hewett; M.; Card; C.; Gasen; M.; Perlman; S.; Verplank (1996) Curricula for HumanComputer Interaction. ACM SIGCHI <http://old.sigchi.org/cdg/> [Consulta: 8/07/2015]

Hurd, Julie M. (2004) Scientific communication: new roles and new players. Science and Technology Libraries, 1/2, (25), 5 - 22.

Hyun, H. K.; Yong, H. K. (2008) Usability study of digital institutional repositories. The Electronic Library. no. 6 (25), 863 - 881.

Souza, S. S.; Barbosa, K. C.; Silva, D. E. L. de S.. (2017) Implantação do repositório institucional do Instituto Federal de Sergipe - RIFS: experiência com o livre acesso, en Encontro de Usuários de Sistemas de Publicação 2017, Brasília (Conferência agendada): IBICT.

Kafure, I. (2004) Usabilidade da imagem na recuperação da informação no catálogo público de acesso em linha. Brasília, Universidad de Brasília.

Kafure, I.; Valencia, M. E.; Rodriguez, P. J.; Florián, B.; Carrillo, J. E.; Solarte, O.; Ciprian, M. (2007) Evaluación de la Usabilidad de la Biblioteca Digital PREDICA, en Seminário Internacional de Bibliotecas Digitais. Anais do Seminário Internacional de Bibliotecas Digitais Brasil. São Paulo: Sistema Integrado de Bibliotecas da USP.

Lazzarin, F. A.; Carneiro; Naiany de Souza; Sousa, E. A. A. de; Sousa; Marckson Roberto Ferreira de. (2012) Da informação à compreensão: reflexões sobre Arquitetura da Informação, Usabilidade e Acessibilidade no campo da Ciência da Informação. Biblionline. João Pessoa, no. esp., 8, pp. 231-244.

Leite, F. C. L. (2009) Como gerenciar e ampliar a visibilidade da informação científica brasileira: repositórios institucionais de Acesso Aberto. Brasília, IBICT.

Machado, K. C.; Vianna, W. B. (2016) Curadoria Digital e Ciência da Informação: correlacões conceituais relevantes para apropriação da informação, en XVII Encontro Nacional de Pesquisa em Pós-Graduação em Ciência da Informação. Salvador - Bahia BA. Anais do XVII Encontro Nacional de Pesquisa em Pós-Graduação em Ciência da Informação. Salvador: GT 1: Estudos Históricos e Epistemológicos da Ciência da Informação. v. 1. 1-15.

Meadows, A. J. (1999) A comunicação científica. Brasília, Briquet de Lemos Livros.

NIELSEN, J. (1995) 10 usability heuristics for user interface design. $<$ http://www.nngroup.com/articles/ten-usability-heuristics/> [Consulta: 01/10/2014]

Norman, D. A. (2008) Design emocional: por que adoramos (ou detestamos) os objetos do dia-a-dia. Rio de Janeiro, Rocco.

Pinheiro, Á. C. de O.; Aguiar Filho, A. S.; Gomes, G. M. R.; Cruz, R. do C.. (2012) Estudos de usos e usuários da informação: uma abordagem em diferentes contextos" Revista Pensar: gestão e administração. 1 (2).

Repositório Institucional da Universidade de Brasília. Downloads and views: time Series (2016). $<\mathrm{http}: / /$ repositorio.unb.br/stats?level=general\&type=access\&page=downviewsseries $>$. [Consulta: 27/01/2016] 
Rocha, S. F. da. (2014) Fatores que influenciam a interação entre usuário e a interface de periódicos científicos eletrônicos: um estudo com autores de artigos no Sistema Eletrônico de Editoração de Revistas (SEER). Brasília, Universidade de Brasília.

Rogers, Y.; Sharp, H.; Preece, J. (2013) Design de interação: além da interação humanocomputador. Porto Alegre, Bookman.

Sebillote, S. (1995) Task TD-5: methodology guide to task analysis with the goal of extracting relevant characteristics for interfaces, Esprit 3 Projet: P6593, "INTUITIVE", INRIA Ronquencourt.

Silva, R. T. S. e. (2014) Estudo de usabilidade em repositório digital de acesso aberto: o caso do RepositóriUM. Guimarães, Portugal, Universidade do Minho.

Sivaji, A.; Nielsen, S. F.; Clemmensen, T. (2017) A Textual Feedback Tool for Empowering Participants in Usability and UX Evaluations, en International Journal of Human-Computer Interaction, 33 (5), 357-370.

Suber, P. (2013) Open Acess overview. <http://legacy.earlham.edu/ peters/fos/overview .htm> [Consulta: 13/10/2014]

Swan, A. (2006) Overview of scholarly communication. <http://eprints.soton.ac.uk/ 262427/1/asj1.pdf> [Consulta: 20/11/2014].

Tsakonas, G.; Papatheodorou, C. (2008) Exploring usefulness and usability in the evaluation of open Access digital libraries. Information Processing \& Management, Filadélfia, 44, 1234-1250.

Veiga, V. S. de O.; Machado, R. R.; Alves, A. da S. (2013) Avaliação de usabilidade em Repositórios Institucionais: o caso da Fiocruz. Congresso Brasileiro de Biblioteconomia, Documentação e Ciência da Informação, XXV. Florianópolis, Brasil. 